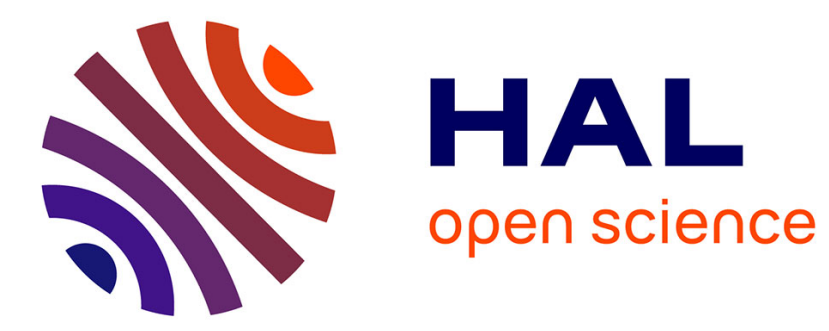

\title{
Femur, tibia and fibula bone templates to estimate subject-specific knee ligament attachment site locations
}

Hélène Pillet, Elena Bergamini, Goulven Rochcongar, Valentina Camomilla, Patricia Thoreux, Philippe Rouch, Aurelio Cappozzo, Wafa Skalli

\section{- To cite this version:}

Hélène Pillet, Elena Bergamini, Goulven Rochcongar, Valentina Camomilla, Patricia Thoreux, et al.. Femur, tibia and fibula bone templates to estimate subject-specific knee ligament attachment site locations. Journal of Biomechanics, 2016, 49 (14), pp.3523-3528. 10.1016/j.jbiomech.2016.09.027 . hal-02471040

\author{
HAL Id: hal-02471040 \\ https://hal.science/hal-02471040
}

Submitted on 7 Feb 2020

HAL is a multi-disciplinary open access archive for the deposit and dissemination of scientific research documents, whether they are published or not. The documents may come from teaching and research institutions in France or abroad, or from public or private research centers.
L'archive ouverte pluridisciplinaire HAL, est destinée au dépôt et à la diffusion de documents scientifiques de niveau recherche, publiés ou non, émanant des établissements d'enseignement et de recherche français ou étrangers, des laboratoires publics ou privés. 


\title{
Femur, tibia and fibula bone templates to estimate subject-specific knee ligament attachment site locations
}

\author{
Hélène Pillet $^{\mathrm{a}, *}$, Elena Bergamini ${ }^{\mathrm{b}}$, Goulven Rochcongar ${ }^{\mathrm{a}, \mathrm{c}}$, Valentina Camomilla ${ }^{\mathrm{b}}$, \\ Patricia Thoreux ${ }^{\mathrm{a}, \mathrm{d}}$, Philippe Rouch ${ }^{\mathrm{a}}$, Aurelio Cappozzo ${ }^{\mathrm{b}}$, Wafa Skalli ${ }^{\mathrm{a}}$ \\ ${ }^{a}$ Arts et Metiers ParisTech, Laboratoire de Biomécanique (LBM)/Institut de Biomécanique Humaine Georges Charpak, Paris, France \\ ${ }^{\mathrm{b}}$ Interuniversity Centre of Bioengineering of the Human Neuromusculoskeletal System (BOHNES), Department of Movement, Human and Health Sciences, \\ University of Rome "Foro Italico", Rome, Italy \\ ' Département d'Orthopédie et Traumatologie, INSERM U1075 COMETE «Mobilité: Attention, Orientation E' Chronobiologie», Université de Caen, France \\ ${ }^{\mathrm{d}}$ Service de Chirurgie Orthopédique et Traumatologique, Hôpital Avicenne - Université Paris 13, Sorbonne Paris Cité, Bobigny, France
}

Keywords:

Knee ligaments

Ligament footprint

Bone templates

Generic model

Scaling

\begin{abstract}
A B S T R A C T
In-vivo estimates of the positions of knee ligament attachment sites are crucial for subject-specific knee modelling. The present study provides template digital models of femur, tibia and fibula that embed the positions of centroids of the origins and insertions of cruciate and collateral ligaments, along with information on their dispersion related to inter-individual variability. By using a shape transformation procedure of choice, these templates can be made to match anatomical information measured on a subject under analysis. Generic bone digital models of the femur, tibia and fibula were first chosen as bone templates. Ligament attachment areas were accurately identified through dissection on the bones of 11 knee specimens, and marked using radio opaque paint. Digital models of these bones embedding the positions of the centroids of the identified ligament attachment areas were thereafter obtained using medical imaging techniques. These centroids were mapped onto the relevant bone template, thus obtaining a cloud of 11 points for each attachment site, and descriptive statistics of the position of these points were thereafter determined. Dispersion of these positions, essentially due to inter-individual variability, was below $6 \mathrm{~mm}$ for all attachment areas. The accuracy with which subject-specific ligament attachment site positions may be estimated using the bone template models provided in this paper was also assessed using the above-mentioned 11 specimens data set, and a leave-one-out cross validation approach. Average accuracy was found to be $3.3 \pm 1.5 \mathrm{~mm}$ and $5.8 \pm 2.9 \mathrm{~mm}$ for femoral and tibial/fibular attachment sites, respectively.
\end{abstract}

\section{Introduction}

Musculoskeletal models of the knee joint are commonly used in several contexts. In human movement analysis, for example, they are included in multi-body optimization methods with the intent of reducing propagation of thigh and shank soft tissue artefacts for the estimation of skeletal kinematics (Clément et al., 2015; Gasparutto et al., 2015). Knee models are used to predict muscle and ligament forces, as well as bone loading, in order to investigate the key factors involved in injury, tissue degeneration or regeneration (Mokhtarzadeh et al., 2014; Yang et al., 2010). Models of the knee joint are also used to design and assess prosthetic implants or to devise custom made patient-specific instruments to improve the accuracy of implants' positioning (Schotanus et al., 2016), as well as in orthopaedic reconstruction procedures (Marra et al., 2015), computer-aided orthopaedic surgery, and in surgery planning (Duarte et al., 2014).

In the above-mentioned contexts, models that represent the anatomy and function of the active and passive components of the knee of a specific living subject are often required (Clément et al., 2015; Marra et al., 2015; Yang et al., 2010). This implies accurately determining the 3-D location of the centroids of knee ligament origin and insertion areas (hereinafter referred to as ligament endpoints), on experimentally acquired or estimated subject-specific bone digital models (Ascani et al., 2015; Li et al., 2009). Ligaments involved are: anterior and posterior cruciate ligaments (ACL and PCL), deep and superficial bundles of the medial collateral ligaments (MCLd and MCLs), and the lateral collateral ligament (LCL).

Extensive literature exists that describes the positions of knee ligament attachment areas as obtained ex-vivo. Most of the studies focused on ACL and PCL (Doi et al., 2009; Guo et al., 2009; Lorenz 
et al., 2009; Osti et al., 2012; Piefer et al., 2012; Zheng et al., 2014), whereas few works have dealt with MCLd, MCLs, and LCL (LaPrade et al., 2007; Liu et al., 2010). All these studies aimed to provide surgeons and clinicians with a detailed, although generic, morphological description of the ligament attachment areas required to perform ligament reconstructions. This information, however, does not allow for the in-vivo identification of subject-specific ligament endpoints.

A number of studies have focused on this latter problem using medical imaging techniques, such as magnetic resonance imaging (MRI) (Ladd et al., 2010), computed tomography (CT) (Ascani et al., 2015; Yoo et al., 2010), or radiography (Wijdicks et al., 2009). However, these techniques are time-consuming, expensive and, when ionizing radiation is involved, invasive for the subjects. Furthermore, results obtained using MRI may be characterized by significant inter- and intra-observer variability, thus leading to errors in the order of $32 \mathrm{~mm}$ (Rachmat et al., 2014), that are exceedingly large when used to generate subject-specific knee models.

A feasible alternative to obtain subject-specific knee ligament endpoints could be based on generic digital models of femur, tibia and fibula (to be considered as bone templates) that embed these endpoints (Bergamini et al., 2011; Hausselle et al., 2014). The bone template may thereafter be submitted to a shape transformation procedure aimed at matching it with anatomical information about the corresponding bone of the subject under analysis, obtained using a non-invasive and doable experimental procedure. In this way subject-specific bone models embedding the relevant ligament endpoints can be obtained (Lew and Lewis, 1977; Lewis et al., 1980). This procedure can be particularly beneficial in clinical contexts where low dose bi-planar X-ray imaging can be routinely used to obtain 3D bone models: enhancing these models by adding the subject-specific ligament endpoint positions, in fact, would allow surgeons to have access to this information without complicating or interfering with the typical procedure.

The purpose of the present study is twofold: first, to provide digital templates of femur, tibia and fibula embedding the 3-D position of knee ligament endpoints, along with information on their dispersion related to inter-individual variability. Second, to validate the use of these generic models to obtain subject-specific knee ligament endpoints. To the aim of obtaining the digital templates, generic bone models of the femur, tibia and fibula were first chosen as bone templates. Lower-limb specimens were dissected and the ligament attachment areas marked and reported on the respective bone models. The relevant bone models embedding the marked ligament attachment areas were matched to the respective bone template and their centroids averaged. To the aim of validating the use of these generic models to obtain subjectspecific knee ligament endpoints, the centroid positions were then estimated, applying a shape transformation procedure to the bone templates and using a leave-one-out cross validation approach, and their accuracy assessed in terms of their Euclidean distance with respect to the measured positions.

\section{Methods}

\subsection{Lower limb specimens and bone digital models}

Eleven lower limb specimens were harvested from subjects aged between 47 and 79 years and fresh frozen. Each specimen included femur, patella, fibula and tibia and intact joint passive structures and exhibited no trauma, surgery or evident deformity. Specimens were thawed at room temperature for $24 \mathrm{~h}$ and three tantalum balls (diameter $=2 \mathrm{~mm}$ ) were pierced into both the distal epiphysis of the femur and the proximal epiphysis of the tibia before beginning the experimental session. These balls were used to register the information obtained by the different instrumentation used in this study, as described in Section 2.4.

Two orthogonal digital radiographs of each specimen (whole femur, tibia and fibula bones, i.e. epiphyses and diaphyses) were simultaneously acquired using a low dose
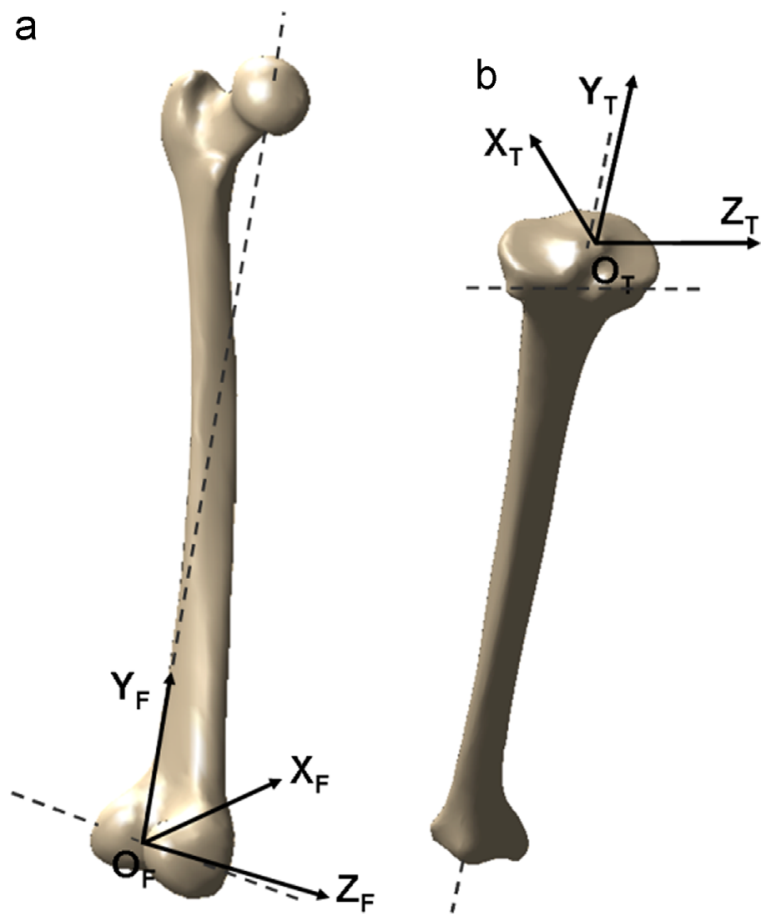

Fig. 1. Femur and tibia bone models and anatomical systems of reference (Schlatterer et al., 2009). (a) Femur: OF (origin): mid-point of the segment joining the centres of the two condylar spheres, obtained by least squares approximation of the posterior portion of the medial and lateral epicondyles; YF: axis going from OF to the centre of the femoral head; ZF: projection onto the plane orthogonal to YF of the segment joining the centres of the two condylar spheres (dashed line); XF: cross product between YF and ZF. (b) Tibia: OT (origin): centroid of the tibial plateaux; YT: axis going from the centroid of the tibial pilon surface to the intersection between the principal inertial axis of the tibial diaphysis (dashed line) and the tibial plateaux surface; ZT: projection onto the plane orthogonal to YT of the segment joining the most posterior points of the tibial plateaux (dashed line); XT: cross product between YT and ZT.

X-ray system (EOS ${ }^{1}$, EOS-imaging, France) and 3-D digital models of the 11 femurs, tibias and fibulas (referred to as specimen-specific bone models) were obtained through a shape reconstruction algorithm described and validated in previous studies (Chaibi et al., 2012; Quijano et al., 2013). This algorithm was selected as representative of the existing shape transformation methods that match generic bone templates to subject-specific bones, relying on partial morphological information of the latter. Briefly, it is based on the following steps: (i) identification and labelling of anatomical landmarks on the radiographic images in order to set the main descriptors of each bone; (ii) generation of a simplified personalized parametric model (SPPM) based on statistical inferences; (iii) deformation of a 3D generic morpho-realistic model towards the SPPM using moving least squares and kriging techniques until the best estimate of the subject-specific bone model is obtained (Fig. 1). The discrepancy between a subject-specific model of a femur or tibia, obtained using this procedure and the relevant CT-scan model, was assessed in previous studies and its 95\% confidence interval was found to be lower than $3.2 \mathrm{~mm}$ in the bone epiphysis areas (Chaibi et al., 2012; Quijano et al., 2013). In addition, the interoperator and landmark identification and its impact on anatomical frame calculation was evaluated by Schlatterer et al. (2009) and Pillet et al. (2014).

The tantalum balls pierced in each bone were manually identified on the radiographs and their coordinates in the X-ray system reference frame obtained. The repeatability of this identification was in the order of $0.1 \mathrm{~m}$ (Dagneaux et al., 2015). Femur and tibia anatomical frames were then defined as suggested in Schlatterer et al. (2009) (Fig. 1). It is worth to underline that the definition of the anatomical systems of reference proposed by Schlatterer et al. (2009) does not require any manual (or virtual) palpation of anatomical landmarks on the femur is, therefore, not prone to the intra- and inter-operator variability associated to the identification of these landmarks (Della Croce et al., 1999) required to define anatomical frames according to the ISB convention (Wu et al., 2002).

\subsection{Identification of ligament attachment areas and registration with the bone digita} models

Each specimen was fully dissected and the attachment areas of the following ligaments were identified by an experienced surgeon: ACL, PCL, MCLd, MCLs, and LCL. A radio opaque paint composed of barium sulphate was used to mark the ligament attachment areas after removing each ligament (Fig. 2a). High resolution CT scans of 
a

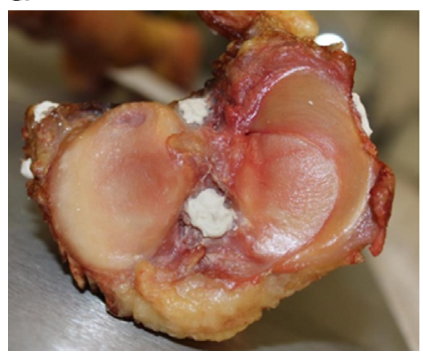

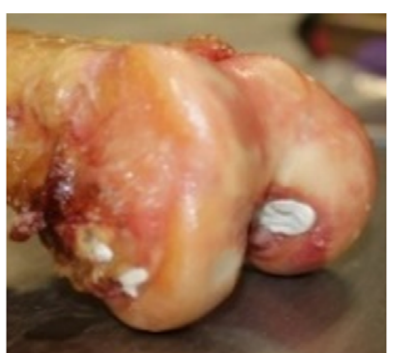

b

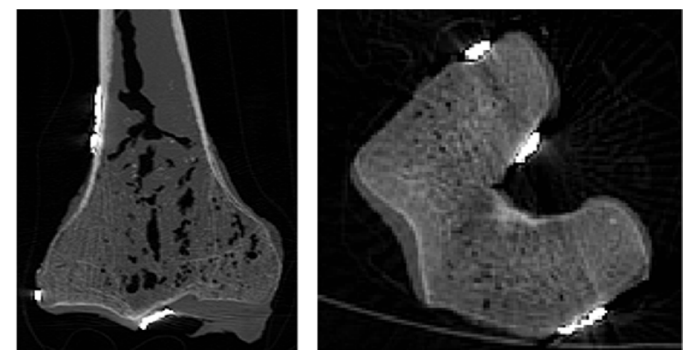

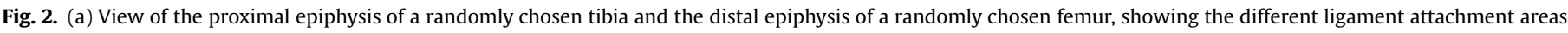

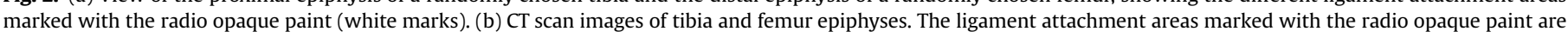
clearly visible in white.
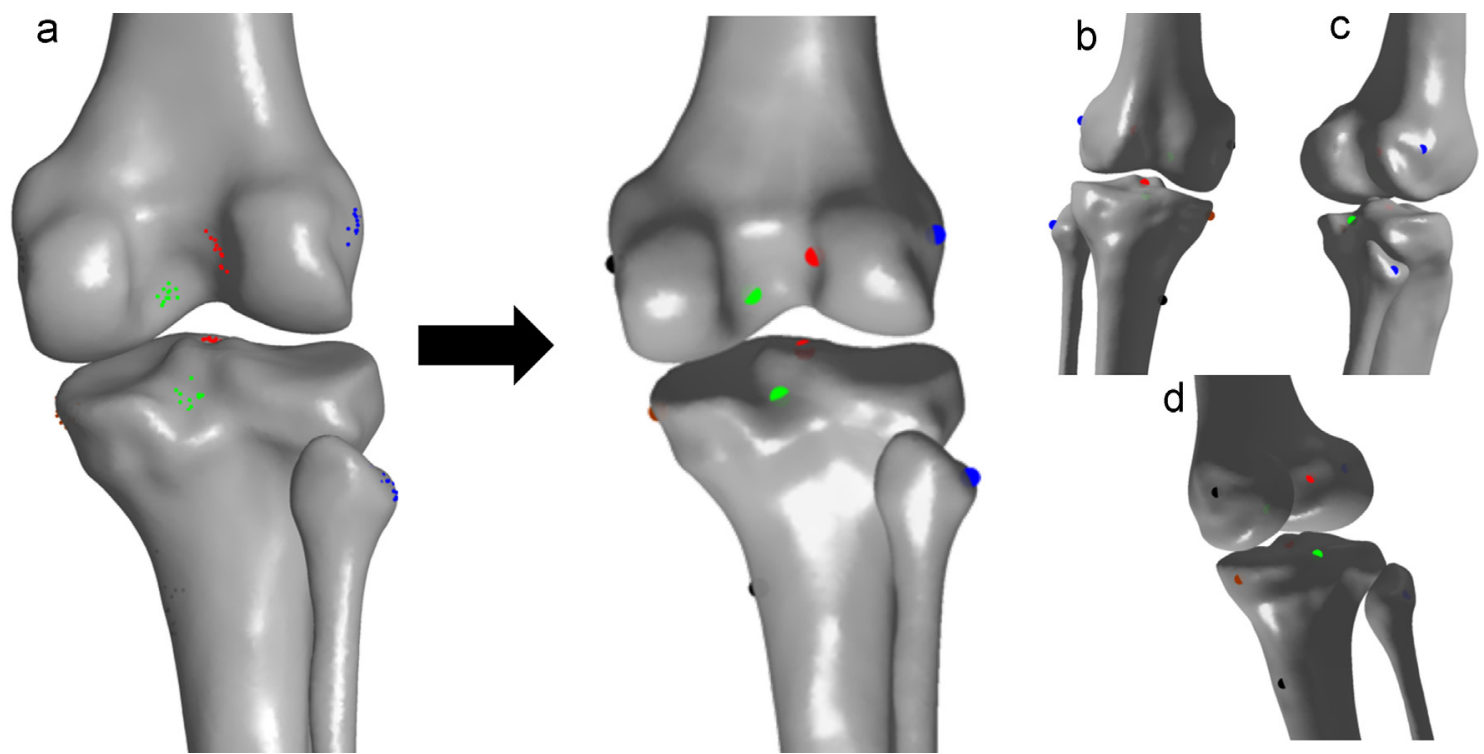

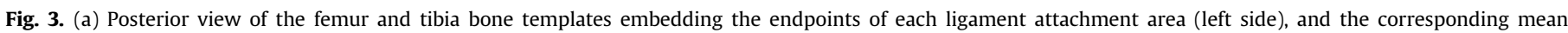
endpoints, $\boldsymbol{p}_{m}$ (right side), (b) anterior view, (c) lateral view, (d) medial view.

each distal femur and proximal tibia/fibula epiphyses were thereafter taken (Fig. 2b) (Philips, Best, The Netherlands; thickness $=0.67 \mathrm{~mm}$, distance $=0.33 \mathrm{~mm}$ ) and digital representations of the ligament attachment areas and tantalum spheres in the CT scan reference frame obtained using the Aviso ${ }^{\mathbb{B}}$ software (Burlington, MA, US). It is worth underlining that in the present study, only the epiphyses of each bone were CT scanned as the rest of the bone was unavailable due to concurrent research studies. Given the accuracy with which the specimen-specific bone models were obtained using the X-ray system (see Section 2.1), we consider this circumstance to have no impact on the end results.

The coordinates of the tantalum spheres, available both in the X-ray and CT scan spaces, were used to register the reference frames of the two systems, and a representation of the attachment areas of each ligament was obtained on the corresponding specimen-specific bone model. It should be noted that, due to the fact that the bone models obtained in the X-ray and CT-scan environments do not perfectly coincide, the registration between the two system reference frames might have as a consequence the fact that the ligament endpoints are rarely located exactly on the bone model surface. The contour points of each area were thus projected onto the surface of the specimen-specific bone model according to the following procedure: first, a least squares plane approximating the attachment area was determined; second, the direction orthogonal to this plane and pointing towards the bone model surface was identified; third, each contour point was projected onto the bone model surface along this direction (the maximal geometric distance between the points of the contours and the bone surface was equal to $3.5 \mathrm{~mm}$ ). Finally, for each specimen-specific bone model, the centroid of each ligament attachment area (for the sake of brevity referred to as true endpoint, $\boldsymbol{p}_{t}$ ) was calculated and its 3-D position expressed with respect to the relevant bone anatomical reference frame (Fig. 1).

\subsection{Digital bone templates}

Generic bone digital models of the femur, tibia and fibula were obtained by reconstruction of CT-scan images of one specimen and chosen as bone templates.
A bone anatomical reference frame was associated with each bone template according to Schlatterer et al. (2009).

\subsection{Ligament endpoints mapping on femur, tibia and fibula bone templates}

To embed the 3-D position of the ligament endpoints in the available generic bone models, each specimen-specific bone model carrying the coordinates of the associated true endpoints, was scaled and deformed using a kriging algorithm (Trochu, 1993) to match the bone templates, either the femur or the tibia/fibula, mentioned in Section 2.3. Through this procedure, the true endpoints were mapped on the relevant bone template. In this way, for each ligament attachment site, the coordinates of 11 endpoints (one for each specimen) were obtained (Fig. 3) and expressed in the bone template anatomical reference frame. The mean of the endpoint coordinates was calculated for each ligament attachment site, and referred to as the template mean endpoint $\left(\boldsymbol{p}_{m}\right)$ (Fig. 3). The dispersion of the 11 endpoint positions around $\boldsymbol{p}_{m}$, was quantified as the standard deviation of the endpoint coordinates for each ligament attachment site. Although this dispersion is due to both experimental errors and inter-specimen variability, the latter phenomenon was expected to play a major role, given the accuracy with which digital bone models are obtained using the selected shape transformation procedure, as reported in Section 2.1.

The information required to represent the 3-D coordinates of the template mean endpoints in the anatomical reference frames defined in the ISB recommendations (Wu et al., 2002), was also obtained. To this aim, a virtual palpation procedure was performed on the bone templates by an expert surgeon and the following bone landmarks were identified and expressed in the template anatomical reference frame chosen for the present study (Schlatterer et al., 2009): medial and lateral epicondyles for the femur; tip of the medial and lateral malleoli, the most medial point on the border of the medial tibial condyle, the most lateral point on the border of the lateral tibial condyle, and the tibial tuberosity for the tibia. In addition, the femoral head was fitted to a sphere, and the 3-D position of its centre computed. 


\subsection{Accuracy in the estimation of subject-specific ligament endpoint positions}

The specimen-specific position of each ligament endpoint was estimated following a leave-one-out cross-validation approach. For each ligament endpoint, one specimen at a time was excluded from the analysis and the mean of the remaining 10 out of 11 endpoints was computed and represented in the bone templates. The shape transformation algorithm previously mentioned (Chaibi et al., 2012; Quijano et al., 2013) was applied to the bone templates embedding the ligament endpoints thus obtained, to match the radiographic images of the left-out specimen. In this way, for each specimen-specific bone model, an estimate of the position of each ligament endpoint, referred to as estimated endpoint $\left(\boldsymbol{p}_{e}\right)$, was obtained and expressed in the relevant bone anatomical reference frame. The accuracy of this estimate was assessed as mean \pm one standard deviation of the Euclidean distance between $\boldsymbol{p}_{t}$ (defined in Section 2.2) and $\boldsymbol{p}_{e}$.

\section{Results}

The 3-D position of each ligament attachment site mean endpoint, $\boldsymbol{p}_{m}$, represented in the bone template anatomical reference frames defined in Fig. 1, is reported in Table 1. The femur, tibia and fibula digital bone templates carrying these 3D coordinates are provided as Supplementary material. The information required to represent these coordinates in the femur and tibia/fibula anatomical reference frames as defined in the ISB recommendations (Wu et al., 2002) are also provided as Supplementary material.

The dispersion of all ligament endpoint positions is presented in Fig. 4. This dispersion was below $4.0 \mathrm{~mm}$ for all ligament endpoints, except for the tibial endpoint of the MCLd $(X$ component $=4.4 \mathrm{~mm})$ and of the MCLs ( $Y$ component $=6.0 \mathrm{~mm}$ ).

The results regarding the accuracy with which subject-specific ligament endpoint positions can be estimated are reported in Table 1. Average accuracy was found to be $3.3 \pm 1.5 \mathrm{~mm}$ and $5.8 \pm 2.9 \mathrm{~mm}$ for femoral and tibial/fibular endpoints, respectively.

\section{Discussion}

In the present study, subject-specific anatomical information, obtained through dissection and imaging techniques from lower limb specimens, was used to obtain reference positions for the origin and insertion centroids (true endpoints) of the cruciate and collateral knee ligaments. The 3-D coordinates of these ligament endpoints were mapped onto digital bone templates of femur, tibia and fibula and made available together with information on their dispersion. The accuracy with which subject-specific ligament endpoint positions may be estimated using these bone

\section{Table 1}

Mean coordinates $\left(\boldsymbol{p}_{m}\right)$ over the 11 ligament endpoint positions as obtained on the femur, tibia and fibula bone templates, for each ligament attachment site, expressed with respect to bone-embedded anatomical reference frames (Schlatterer et al., 2009). The Euclidean distance between the estimated $\left(\boldsymbol{p}_{e}\right)$ and true $\left(\boldsymbol{p}_{t}\right)$ endpoint positions is also reported in terms of mean and one standard deviation.

\begin{tabular}{|c|c|c|c|c|c|c|}
\hline & \multirow[t]{2}{*}{ Ligament } & \multicolumn{3}{|c|}{$\boldsymbol{p}_{m}(\mathrm{~mm})$} & \multirow{2}{*}{$\begin{array}{l}\begin{array}{l}\text { Accuracy assessment } \\
(\mathrm{mm})\end{array} \\
\begin{array}{l}\text { Euclidean distance } \\
\text { between } \boldsymbol{p}_{e} \text { and } \boldsymbol{p}_{t}\end{array}\end{array}$} & \\
\hline & & $X$ & $Y$ & $Z$ & & \\
\hline \multirow[t]{4}{*}{ Femur } & $\mathrm{ACL}$ & -5.0 & 3.6 & 11.9 & 3.9 & \pm 1.6 \\
\hline & PCL & 6.1 & -7.8 & -7.8 & 2.7 & \pm 1.5 \\
\hline & MCL & 3.4 & 2.4 & -41.7 & 3.6 & \pm 1.7 \\
\hline & LCL & 1.8 & 6.5 & 42.2 & 3.0 & \pm 1.3 \\
\hline \multirow[t]{5}{*}{ Tibia/Fibula } & $\mathrm{ACL}$ & 5.1 & 4.4 & 0.5 & 3.6 & \pm 1.2 \\
\hline & PCL & -13.7 & -5.6 & -3.1 & 3.9 & \pm 0.7 \\
\hline & MCLd & 2.7 & -9.5 & -38.4 & 6.7 & \pm 3.6 \\
\hline & MCLs & 11.7 & -58.5 & -18.0 & 9.5 & \pm 7.0 \\
\hline & $\mathrm{LCL}$ & -16.5 & -29.6 & 45.4 & 5.2 & \pm 2.0 \\
\hline
\end{tabular}

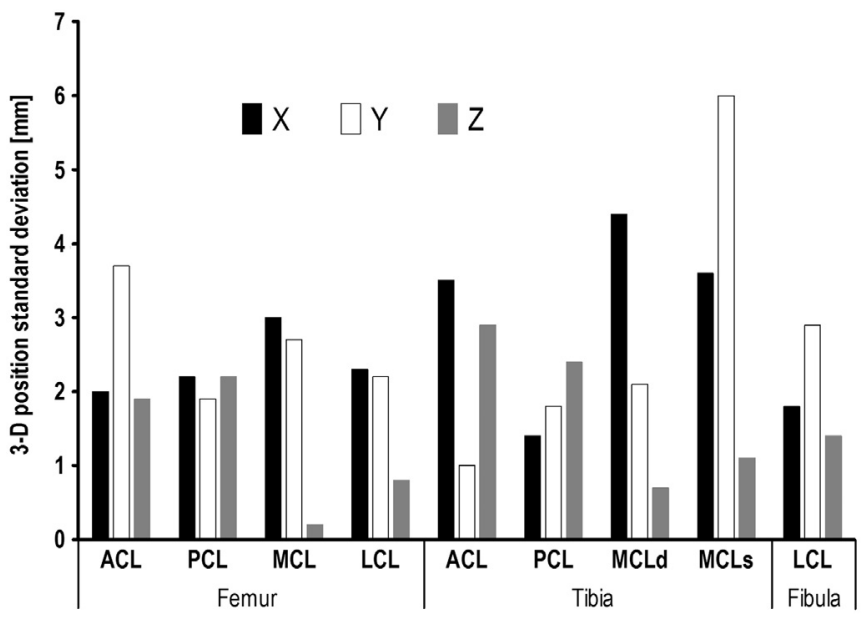

Fig. 4. Dispersion (one standard deviation of the $X, Y$, and $Z$ coordinates) of the eleven ligament endpoint positions as obtained, for each attachment site, on the femur and tibia/fibula bone templates.

templates was assessed in terms of Euclidean distance between estimated and true endpoint positions.

For what concerns the femur, the positions of all subjectspecific ligament endpoints were estimated with average errors smaller than $4.0 \mathrm{~mm}$. Slightly lower accuracy was obtained for the positions of the endpoints on the tibial/fibular bones, particularly for the collateral ligaments, for which errors increased to $5.2 \mathrm{~mm}$, $6.7 \mathrm{~mm}$, and $9.5 \mathrm{~mm}$ for the LCL, the deep (MCLd) and superficial (MCLs) bundles of the MCL, respectively. These values are in agreement with the study of Ascani et al. (2015), which reports maximal errors of $8.0 \mathrm{~mm}$ (for the femoral attachment sites) and $11.3 \mathrm{~mm}$ (for the tibial/fibular attachment sites) in estimating subject-specific ligament endpoints from CT images using MRI data as a reference. It must be noted that current results cannot easily be compared with most of the existing literature estimating subject-specific ligament endpoints in-vivo using imaging techniques (Ladd et al., 2010; Wijdicks et al., 2009; Yoo et al., 2010), since these studies provide the ligament attachment area locations only with respect to anatomical landmarks that are commonly used by surgeons during knee surgery and do not provide information about the accuracy with which these locations are obtained.

The greater errors obtained in estimating the collateral ligament endpoint positions on the tibia/fibula relative to the femur are probably due to the much greater dispersion of the former (up to $6.0 \mathrm{~mm}$ ) with respect to the latter (up to $3.0 \mathrm{~mm}$, Fig. 4). This dispersion can be hypothesized as due to a correspondingly large inter-individual variability of these attachment sites (Liu et al., 2010), and cannot be taken into account when using the template mean endpoint $\boldsymbol{p}_{m}$ to estimate the subject-specific ligament endpoint positions. The relevance of the reported errors, however, depends on how the estimated information is used, and must be evaluated in terms of impact on the parameters considered for the application of interest (i.e. ligament length variation, ligament strain, or tunnel location during ligament reconstruction).

It is worth noting that, in the present study, the anatomical information necessary to match the femur and tibia/fibula bone templates to specimen-specific bone models was obtained using a low dose biplanar X-ray system, which is an imaging technique that can be used in-vivo and, thus, often employed in the clinical context. For those contexts where imaging techniques cannot be used, the necessary anatomical information may be derived by acquiring partial features of the bones through stereophotogrammetry and manual palpation of superficial landmarks selected over the areas of the bone covered with a thin layer of soft 
tissue (Donati et al., 2008, 2007; Luo et al., 2009), therefore without the use of any medical imaging techniques. The accuracy with which subject-specific ligament endpoint positions are estimates would be a direct consequence of the characteristics of the method used for subject-specific bone model estimation and may be lower than that reported in the present study, especially for subjects whose bone epiphyses morphology is considerably altered by pathological conditions such as, for example, osteoarthritis. Whether this lower accuracy is acceptable depends, again, on the specific application.

As concerns the shape transformation algorithm, the method used in the present study, is the one proposed by Chaibi et al. (2012) and Quijano et al. (2013) and its accuracy was quantified in estimating the specimen-specific location of ligament endpoints. This method can be considered as representative of the existing shape transformation algorithms based on non-homogenous scaling (Audenino et al., 1996; Brand et al., 1982; Gunay et al., 2007; Lewis et al., 1980) which must be chosen depending on both theoretical and practical constraints.

In this work, reference values for the ligament endpoint positions were obtained by dissecting each specimen, marking the ligament attachment areas with radio opaque paint, and acquiring CT images of each bone epiphysis. When ex-vivo studies are considered, this methodology may be a valid alternative to using stereophotogrammetry or MRI data, whose accuracy has been recently undermined (Rachmat et al., 2014). In this respect, it should also be noted that the combined use of computed tomography and stereoradiography allowed us to obtain specimenspecific high-resolution information about both ligament attachment areas and digital bone models in a reasonable amount of time (about $10 \mathrm{~min}$ to reconstruct the attachment areas and the femur, tibia and fibula digital models for each specimen). Last but not least, the availability of femur and tibia/fibula templates embedding information from 11 different specimens, provided an indication of the inter-individual variability of each ligament endpoint position that, although affected by similar measurement errors, is not affected by scale-effects due to different bone anthropometries.

The following limitations warrant acknowledgement in consider the generalizability of current results: (a) the limited number of specimens which, however, is comparable with that considered in the majority of published studies focusing on similar topics and using ex-vivo approaches; (b) the dependency of the reported accuracy on how the anatomical information necessary to match the femur and tibia/fibula bone templates to subject-specific bone models are obtained, as well as on the selected shapetransformation algorithm.

In conclusion, the femur and tibia/fibula bone templates made available in this study can be used to estimate reasonably accurate subject-specific positions of each ligament endpoint, once sufficient anatomical information of the bone of a given individual is available, and without necessarily requiring the use of imaging techniques. The obtained information may be particularly useful to develop individualized and realistic musculoskeletal knee models for application in research context. Moreover, when relying on medical imaging, they can be used in a clinical context, for example, for the planning of the ACL reconstruction surgery, where the one of the first cause of failure is the malposition of the femoral tunnel (Trojani et al., 2011).

\section{Conflict of interest statement}

The authors declare they have no financial or personal relationships with other people or organization that could inappropriately influence their work.

\section{Acknowledgement}

The contribution of Ann-Laure Pollastri and of Louis Dagneaux is gratefully acknowledged. The authors also thank the ParisTech BiomecAM chair program on subject-specific musculoskeletal modelling, with the support of COVEA and Société Générale.

\section{References}

Ascani, D., Mazzà, C., De Lollis, A., Bernardoni, M., Viceconti, M., 2015. A procedure to estimate the origins and the insertions of the knee ligaments from computed tomography images. J. Biomech. 48, 233-237.

Audenino, A.L., Zanetti, E.M., Calderale, P.M., 1996. Radiograph processing of bone remodelling for quantitative assessment. Med. Eng. Phys. 18, 382-389.

Bergamini, E., Pillet, H., Hausselle, J., Thoreux, P., Guerard, S., Camomilla, V., Cappozzo, A., Skalli, W., 2011. Tibio-femoral joint constraints for bone pose estimation during movement using multi-body optimization. Gait Posture 33, 706-711.

Brand, R.A., Crowninshield, R.D., Wittstock, C.E., Pedersen, D.R., Clark, C.R., van Krieken, F.M., 1982. A model of lower extremity muscular anatomy. J. Biomech. Eng. 104, 304-310.

Chaibi, Y Cresson, T. Aubert, B., Hausselle, J., Neyret, P., Hauger, O., de Guise, J.A Skalli, W., 2012. Fast 3D reconstruction of the lower limb using a parametric model and statistical inferences and clinical measurements calculation from biplanar X-rays. Comput. Methods Biomech. Biomed. Eng. 15, 457-466.

Clément, J., Dumas, R., Hagemeister, N., de Guise, J.A., 2015. Soft tissue artifact compensation in knee kinematics by multi-body optimization: performance of subject-specific knee joint models. J. Biomech. 48, 3796-3802.

Dagneaux, L., Thoreux, P., Eustache, B., Canovas, F., Skalli, W., 2015. Sequential 3D analysis of patellofemoral kinematics from biplanar X-rays: in vitro validation protocol. Orthop. Traumatol. Surg. Res. 101, 811-818.

Della Croce, U., Cappozzo, A., Kerrigan, D.C., 1999. Pelvis and lower limb anatomical landmark calibration precision and its propagation to bone geometry and joint angles. Med. Biol. Eng. Comput. 37, 155-161.

Doi, M., Takahashi, M., Abe, M., Suzuki, D., Nagano, A., 2009. Lateral radiographic study of the tibial sagital insertions of the anteromedial and posterolateral bundles of human anterior cruciate ligament. Knee Surg. Sports Traumatol. Arthrosc. 17, 347-351.

Donati, M., Camomilla, V., Vannozzi, G., Cappozzo, A., 2007. Enhanced anatomical calibration in human movement analysis. Gait Posture 26, 179-185.

Donati, M., Camomilla, V., Vannozzi, G., Cappozzo, A., 2008. Anatomical frame identification and reconstruction for repeatable lower limb joint kinematics estimates. J. Biomech. 41, 2219-2226.

Duarte, R.M., Ferreira, N.V., Oliveira, A.M., Fonseca, F.P., Vieira-Silva, M., CorreiaPinto, J., 2014. Benefits of radial distortion correction in arthroscopic surgery: a first experimental study on a knee model. Int. J. Med. Robot.

Gasparutto, X., Sancisi, N., Jacquelin, E., Parenti-Castelli, V., Dumas, R., 2015. Validation of a multi-body optimization with knee kinematic models including ligament constraints. J. Biomech., 1-6.

Gunay, M., Shim, M.-B., Shimada, K., 2007. Cost- and time-effective three-dimensional bone-shape reconstruction from X-ray images. Int. J. Med. Robot. Comput. Assist. Surg. 3, 323-335.

Guo, L., Yang, L., Wang, A.M., Wang, X.Y., Dai, G., 2009. Roentgenographic measurement study for locating femoral insertion site of anterior cruciate ligament: a cadaveric study with X-Caliper. Int. Orthop. 33, 133-137.

Hausselle, J., Assi, A., El Helou, A., Jolivet, E., Pillet, H., Dion, E., Bonneau, D., Skalli, W., 2014. Subject-specific musculoskeletal model of lower limb in a lying and standing position. Comput. Methods Biomech. Biomed. Eng. 17, 480-487.

Ladd, P.E., Laor, T., Emery, K.H., Salisbury, S.R., Parikh, S.N., 2010. Medial collateral ligament of the knee on magnetic resonance imaging: does the site of the femoral origin change at different patient ages in children and young adults? J. Pediatr. Orthop. 30, 224-230.

LaPrade, R.F., Engebretsen, A.H., Ly, T.V., Johansen, S., Wentorf, F. a, Engebretsen, L., 2007. The anatomy of the medial part of the knee. J. Bone Jt. Surg. Am. 89, 2000-2010.

Lew, W.D., Lewis, J.L., 1977. An anthropometric scaling method with application to the knee joint. J. Biomech. 10, 171-181.

Lewis, J.L., Lew, W.D., Zimmerman, J.R., 1980. A nonhomogeneous anthropometric scaling method based on finite element principles. J. Biomech. 13, 815-824.

Li, K., O’Farrell, M., Martin, D., Kopf, S., Harner, C., Zhang, X., 2009. Mapping ligament insertion sites onto bone surfaces in knee by co-registration of CT and digitization data. J. Biomech. 42, 2624-2626. 
Liu, F., Yue, B., Gadikota, H.R., Kozanek, M., Liu, W., Gill, T.J., Rubash, H.E., Li, G., 2010. Morphology of the medial collateral ligament of the knee. J. Orthop. Surg. Res. 5, 69.

Lorenz, S., Elser, F., Brucker, P.U., Obst, T., Imhoff, A.B., 2009. Radiological evaluation of the anterolateral and posteromedial bundle insertion sites of the posterior cruciate ligament. Knee Surg. Sports Traumatol. Arthrosc. 17, 683-690.

Luo, W., Stanhope, S.J., Sheehan, F.T., 2009. Using two palpable measurements improves the subject-specific femoral modeling. J. Biomech. 42, 2000-2005.

Marra, M.A., Vanheule, V., Fluit, R., Koopman, B.H.F.J.M., Rasmussen, J., Verdonschot, N., Andersen, M.S., 2015. A subject-specific musculoskeletal modeling framework to predict in vivo mechanics of total knee arthroplasty. J. Biomech. Eng. 137, 020904.

Mokhtarzadeh, H., Perraton, L., Fok, L., Muñoz, M. a, Clark, R., Pivonka, P., Bryant, A.L., 2014. A comparison of optimisation methods and knee joint degrees of freedom on muscle force predictions during single-leg hop landings. J. Biomech. 47, 2863-2868.

Osti, M., Tschann, P., Künzel, K.H., Benedetto, K.P., 2012. Anatomic characteristics and radiographic references of the anterolateral and posteromedial bundles of the posterior cruciate ligament. Am. J. Sports Med. 40, 1558-1563.

Piefer, J.W., Pflugner, T.R., Hwang, M.D., Lubowitz, J.H., 2012. Anterior cruciate ligament femoral footprint anatomy: systematic review of the 21 st century literature. Arthroscopy 28, 872-881.

Pillet, H., Sangeux, M., Hausselle, J., El Rachkidi, R., Skalli, W., 2014. A reference method for the evaluation of femoral head joint center location technique based on external markers. Gait Posture 39, 655-658.

Quijano, S., Serrurier, A., Aubert, B., Laporte, S., Thoreux, P., Skalli, W., 2013. Threedimensional reconstruction of the lower limb from biplanar calibrated radiographs. Med. Eng. Phys. 35, 1703-1712.

Rachmat, H.H., Janssen, D., Zevenbergen, W.J., Verkerke, G.J., Diercks, R.L., Verdonschot, N., 2014. Generating finite element models of the knee: how accurately can we determine ligament attachment sites from MRI scans? Med. Eng. Phys. 36, 701-707.
Schlatterer, B., Suedhoff, I., Bonnet, X., Catonne, Y., Maestro, M., Skalli, W., 2009. Skeletal landmarks for TKR implantations: evaluation of their accuracy using EOS imaging acquisition system. Orthop. Traumatol. Surg. Res. 95, 2-11.

Schotanus, M.G., Boonen, B., Kort, N.P., 2016. Patient specific guides for total knee arthroplasty are ready for primetime. World J. Orthop. 7, 61-68.

Trochu, F., 1993. A contouring program based on dual kriging interpolation. Eng. Comput. 9, 160-177.

Trojani, C., Sbihi, A., Djian, P., Potel, J.-F., Hulet, C., Jouve, F., Bussière, C., Ehkirch F.-P., Burdin, G., Dubrana, F., Beaufils, P., Franceschi, J-P., Chassaing, V., Colombet, P., Neyret, P., 2011. Causes for failure of ACL reconstruction and influence of meniscectomies after revision. Knee Surg. Sports Traumatol. Arthrosc. 19 196-201.

Wijdicks, C. a, Griffith, C.J., LaPrade, R.F., Johansen, S., Sunderland, A., Arendt, E. a, Engebretsen, L., 2009. Radiographic identification of the primary medial knee structures. J. Bone Jt. Surg. Am. 91, 521-529.

Wu, G., Siegler, S., Allard, P., Kirtley, C., Leardini, A., Rosenbaum, D., Whittle, M., D'Lima, D.D., Cristofolini, L., Witte, H., Schmid, O., Stokes, I., 2002. ISB recommendation on definitions of joint coordinate system of various joints for the reporting of human joint motion-part I: ankle, hip, and spine. J. Biomech. 35 543-548.

Yang, Z., Wickwire, A.C., Debski, R.E., 2010. Development of a subject-specific mode to predict the forces in the knee ligaments at high flexion angles. Med. Biol. Eng. Comput. 48, 1077-1085.

Yoo, Y.-S., Jeong, W.-S., Shetty, N.S., Ingham, S.J.M., Smolinski, P., Fu, F., 2010 Changes in ACL length at different knee flexion angles: an in vivo biomechanical study. Knee Surg. Sports Traumatol. Arthrosc. 18, 292-297.

Zheng, L., Harner, C.D., Zhang, X., 2014. The morphometry of soft tissue insertions on the tibial plateau: data acquisition and statistical shape analysis. PLoS One 9 e96515. 